\title{
Perceptual-cognitive expertise in combat sport: from scientific research to training
}

\author{
Óscar MARTÍNEZ DE QUEL ${ }^{* 1}, \&$ Simon J. BENNETT2 2 \\ ${ }^{1}$ Faculty of Education - University Complutense of Madrid (Spain) \\ ${ }^{2}$ Research Institute for Sport and Exercise Sciences- Liverpool John Moores University (UK)
}

\section{Introduction}

Performance in combat sports has been widely studied from the point of view of physiology, anthropometrics or biomechanics. Thus, conditional training is usually based on physiological research and techniques are evaluated from a biomechanical perspective. However, combat sport experts also differ from novices in their psychological characteristics. In fact, many trainers suggest the difference between a good athlete and a champion is the ability to make the right technique at the right moment, and that this requires expert perception, cognition, decision making, and tactical awareness. Given the importance of psychological characteristics, it is somewhat surprising that they are usually trained using methods based on the experience of the coach more rather than scientific research. Our aim, therefore, was to review relevant scientific literature on combat sports expertise in a selection of perceptual-cognitive variables and then to provide some guidelines for training.

\section{Research in perceptual-cognitive expertise}

Scientific research in combat sport athletes (boxing, fencing, karate, taekwondo) has given some interesting outcomes. The large number of studies on Reaction Time (RT) has shown inconsistent results, with some evidence that expert athletes have shorter RT but other studies showing that RT is not a predictor of competition success (Martinez de Quel, Bennett, López, Zapico, \& Saucedo, 2015). As the RT task becomes more sport-specific (i.e.: stimulus and response are similar to those in combat), differences are more evident between experts and novices. However, this is often because experts show a good capacity for anticipation and are able to identify an attack in the very first moments (Rosalie \& Muller, 2013). This superior anticipation is helped by their visual behavior, such as maintaining gaze in a central part of the opponent's body (head and trunk). In this way, gaze acts as a visual pivot from where the expert combat athlete can pick-up the information needed to create an appropriate response (Ripoll, Kerlirzin, Stein, \& Reine, 1995), as well as gain knowledge of situational probabilities (Milazzo, Farrow, Ruffault, \& Fournier, 2015), which is linked to tactical training.

\section{Application to training}

The first application is to train coupled action and perception as much as we can such that athletes are exposed to the same constraints as in a combat. This can be achieved by competition, sparring, attack-defense exercises, overloading perceptual system using a task with two attackers versus one defender or wearing liquid crystal glasses that switch from a transparent to opaque state.

From the point of view of the fighter who responds to an attack by performing a defense, anticipated-counterattack, displacement or dodge, it is important to do the following: maintain gaze 
in the center of the opponent's body (head, trunk) most of the time; try to obtain prior information from the opponent body and the environment (score, time to the end, situation on the combat field, more usual techniques of the opponent) to estimate the probabilities of the opponent's actions; adjust the distance to the opponent as a function of the time needed to respond; maintain an initial position (on guard stance) that allows quick reactive movement; create responses that could be adapted to a potential change in the attack or a response useful for many attacks (i.e. step backward could be useful for many attacks); change reactions to avoid the second intention of attacker; etc.

From the point of view of attacker, it is clearly important to try to minimize the ability of the defender to anticipate and this should be taking into account when teaching and evaluating technique. Thus, some options available to the attacker are to: make unpredictable and fast attacking moves; approach the opponent in such a way that there is less time to perceive and act upon the attack; minimize previous movement that can be used by the opponent to anticipate the subsequent attack or even make other movement that give a false impression about the impending attack; and perform attacks that cannot be identified until the very end (i.e. techniques which start from the same position and the same direction and change later). Because the roles of attacker and defender are overlapped in combat sports, consideration of rapid changes of condition should be trained (i.e., to change from attack to defense or vice versa, or even to attack and defend at same time). In all these cases, it is critical that the action takes into account the distance between opponents.

In conclusion, trainers could use scientific knowledge in perceptual-cognitive expertise when designing practice in order to improve defense or attack techniques and tactics.

\section{References}

Martinez de Quel, O., Bennett, S. J., López, E., Zapico, A.G., \& Saucedo, F. (2015). Choice reaction time is not related to competition success in karate combat. European Journal of Human Movement, $35,41-50$.

Milazzo, N., Farrow, D., Ruffault, A., \& Fournier, J.F. (2015). Do karate fighters use situational probability information to improve decision-making performance during on-mat tasks? Journal of Sports Sciences, 16(3), 243-249.

Ripoll, H., Kerlirzin, Y., Stein, J.-F., \& Reine, B. (1995). Analysis of information processing, decision making, and visual strategies in complex problem solving sport situations. Human Movement Science, 14(3), 325-349.

Rosalie, S. M., \& Muller, S. (2013). Timing of in situ visual information pick-up that differentiates expert and near-expert anticipation in a complex motor skill. Quarterly journal of experimental psychology, 66(10), 1951-62.

Key words: Reaction time; perception; tactics; martial arts; combat sports. 